\title{
Medical Innovation Taking Form: 3D Printing at The Ottawa Hospital
}

\author{
Jonathan R. Whelan ${ }^{1}$, Alexander J. Lingley ${ }^{1}$, Olivier Miguel ${ }^{2}$, Adnan Sheikh ${ }^{3}$ \\ ${ }^{1}$ Faculty of Medicine, University of Ottawa, Ontario, Canada \\ ${ }^{2}$ Ottawa Hospital Researh Institute, Ontario, Canada \\ ${ }^{3}$ Department of Radiology, The Ottawa Hospital, Ontario, Canada
}

ABSTRACT

Three-dimensional (3D) printing is an emerging medical technology with capacity to revolutionize multitude aspects of clinical care and medical education. This commentary highlights The Ottawa Hospital Medical 3D Printing Program, a leading Canadian site for the manufacturing of 3D-printed clinical solutions. In Ottawa, 3D printing has already been employed to optimize preoperative surgical planning, facilitate communication with patients, and enhance medical learning and outreach efforts. Rapid advances in manufacturing technology are poised to further expand current medical applications of 3D printing nationwide.

\section{RÉSUMÉ}

L'impression tridimensionnelle (3D) est une technologie médicale émergente avec la capacité de révolutionner plusieurs aspects des soins cliniques et de l'éducation médicale. Ce commentaire surligne le programme d'impression médicale 3D de I'Hôpital d'Ottawa, un site canadien éminent pour la fabrication de solutions cliniques imprimées en 3D. À Ottawa, l'impression 3D a déjà été employée pour optimiser la planification chirurgicale préopératoire, pour faciliter la communication avec les patients, et pour améliorer l'apprentissage médical et la portée. Des avances rapides en des technologies de fabrication sont prêtes à développer de plus les applications médicales actuelles d'impression 3D à l'échelle nationale.

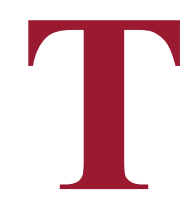

hree-dimensional (3D) printing describes a group of manufacturing techniques permitting digital blueprints to be translated into the synthesis of 3D objects. The first form of 3D printing, originally coined stereolithography, was patented in 1986 (1). Since that time, rapid advances in manufacturing instrumentation and materials technology has led to 3D printers with capabilities approaching or surpassing those of traditional manufacturing techniques. Indeed, modern 3D printing enables custom objects, composed of a wide variety of materials, to be synthesized with acute accuracy and precision, often with significant time- and cost-savings (2). These features of 3D printing lend themselves well to applicability in medicine, where vast patient diversity and rapid technological advancement demand flexible and expedient material solutions.

3D-printed objects have demonstrated utility in a widening variety of clinical applications over the last two decades $(3,4)$, often with significant cost and/or time savings compared to traditional approaches (5). In clinical settings, 3D printing technology has been employed to facilitate surgical planning, fabrication of surgical tools, medical and patient education, as well as bioprinting of synthetic tissues, amongst other applications (6). The continual widening of these applications is expected to push the 3D printing medical devices market value to upwards of $\$ 1.88$ billion USD by 2022 (7).

Consequent to an evolving interest in the clinical value of 3D printing, specialized manufacturing teams and facilities are increasingly incorporated into the hospital and medical education milieu. One such example of this collaboration between engineering and medicine is The Ottawa Hospital (TOH) Medical 3D Printing Program. Formally established in 2017, TOH's 3D Printing Program acts as a central hub, integrating contributions from a dynamic group of teams at $\mathrm{TOH}$ and the University of Ottawa Faculty of Medicine. Under the leadership of radiologists Dr. Frank Rybicki and Dr. Adnan Sheikh, the TOH Medical 3D Printing Program aims to explore means by which advances in 3D printing can be harnessed to tailor novel clinical solutions and innovations in medical education. TOH's 3D Printing Program is Canada's first hospital-integrated 3D medical printing institution and serves an increasingly important component of medical care

Keywords: 3D Print; Medical Innovation; Anatomical Model; Medical Education 
for patients at $\mathrm{TOH}(8)$.

In this commentary, we focus on the current practices and future directions of the $\mathrm{TOH}$ Medical 3D Printing Program. Through description of specific applications and pilot educational programs, we aim to convey how 3D manufacturing is currently revolutionizing clinical care and medical education at $\mathrm{TOH}$, as well as provide a prelude for notable innovations on the horizon.

\section{SURGICAL APPLICATIONS OF 3D ANATOMICAL MODELING}

High-acuity radiological imaging permits the physical recapitulation of patient-specific 3D-printed anatomical models. Through this process, radiologists and surgeons are able to visualize and physically engage with anatomical structures in situ, just as they would encounter them during surgery. Patient-specific 3D anatomical models may therefore permit a degree of surgical planning and preparedness not otherwise attainable with traditional radiological imaging. For this reason, preoperative anatomical models are one of the more prevalent clinical applications of the TOH 3D Printing Program. Customized 3D-printed structures have been used preoperatively at $\mathrm{TOH}$ to model complex fractures of the pelvis, reproduce neoplastic masses of varying origin, and to instruct the dimensions of physical implants so as to ensure optimization with biological structures during reconstructive surgery. In each case, 3D models may have facilitated a greater awareness of patient anatomy prior to surgery, potentially benefitting patient care by improving surgical precision, mitigating the risk of surgical error, and decreasing the duration of surgery. Indeed, in a recent systematic review, a majority of studies described a significant reduction in operating time, improved anatomical representation, and enhanced surgical outcomes when similar anatomical models have been employed at other centers (9).

According to physicians involved in previous surgical applications of 3D printing, the technology offers a tangible clinical benefit. Dr. Joel Werier, an Orthopaedic Surgeon and Oncologist at TOH has employed 3D-printed models to plan complex oncology surgeries since the TOH 3D Printing Program was initiated. He recently remarked that "3D printing is revolutionizing the way we look at anatomy" (10). He added, "it adds another perspective to how we view tumours, how we plan our surgery techniques, and our ability to offer precision surgery" (10).
In addition to their use in preoperative planning, 3D-printed models can allow $\mathrm{TOH}$ patients an opportunity to gain unique insight into their illness. For example, Dr. Sukhbir (Sony) Singh, of the TOH Surgical Gynecology Department, sought the aid of the 3D printing program for assistance with a complex uterine abdominal myomectomy surgical case requiring the excision of forty-eight subserosal and two submucosal fibroids (11). The goal of the procedure was to preserve the patient's uterus, permitting a chance of future pregnancy that would not have been possible following an invasive hysterectomy. Prior to the surgery, Dr. Singh presented the 3D model to his patient so as to explain her upcoming procedure in the context of her own anatomy. Patient understanding of their health status and of proposed surgical procedures is important to ensure the integrity of patient's surgical decision-making (12). We postulate that incorporating anatomy or procedure-specific 3D models in the patient-physician interaction, such as in this case, may help to better inform patients and to promote accurate expectations of their postoperative course.

\section{APPLICATIONS IN MEDICAL EDUCATION}

With few exceptions, anatomical knowledge forms the groundwork from which all clinical decisions are based. Traditional teaching in medical anatomy has emphasized the conjunctive use of two-dimensional (2D) images and cadaveric-based learning. However, there are limitations associated with each of these educational models. For instance, 2D images fall short in engendering an understanding of depth, dimension, or scale amongst learners. While considered a valuable learning resource, cadaveric simulation is limited by high cost, lack of availability, and ethical issues (13). Adjunctive use of 3D-printed educational models in medical education can help address these limitations and optimize anatomical learning. 3D models allow learners to appreciate those details of anatomical structures that would otherwise be obscured by 2D images. By providing both normal and pathology-specific anatomical models, 3D printing also promotes an awareness of anatomical variants and abnormalities less amenable to cadaveric simulation (14).

In collaboration with the TOH 3D Printing Program, researchers at the University of Ottawa are actively exploring the utility of 3D-printed anatomical models in anatomical education. The tracheobronchial tree, housed within the lungs, is a complex biological structure that is difficult to appreciate in $2 \mathrm{D}$ images or visualize in the cadaveric laboratory. 3D-printed bronchial structures, however, provide healthcare practitioners and 
trainees an opportunity to physically interact with respiratory anatomy, potentially allowing for a greater understanding of normal and pathologic respiratory physiology. Radiologist Dr. Carolina Souza is currently leading an investigation aiming to examine the utility of a 3D-printed tracheobronchial medical education model in promoting accurate interpretation of pulmonary computed tomography (CT) scans amongst medical students and residents. Their group hypothesizes that the 3D model will provide an opportunity for superior learning as compared to traditional 2D textbook images (unpublished). Innovation in clinical teaching, however, is but one example of the power of 3D printing to inspire a fascination of medicine and science. 3D-printed biological structures also provide members of the public an opportunity to interact and engage with medical concepts that would otherwise be limited to healthcare trainees. The TOH 3D Printing Program recently launched a permanent exhibit showcasing 3D-printed anatomical models at the Canada Science \& Technology Museum. The exhibit consists of two parts: a display of mounted 3D-printed anatomical structures including models of the heart, lungs, pelvis, and liver as well as a hands-on exploration area where 3D-printed anatomical models are visually concealed but identified through touch \& feel. This has become one of the most popular exhibits at the Canada Science \& Technology Museum, which welcomes 650,000 visitors annually (15).

3D-printed models have also been integral in another outreach initiative recently launched at the Canada Science \& Technology Museum. Medical Curiosity on Stage, a series of interactive and captivating presentations hosted by University of Ottawa medical students, seeks to immerse young children and adolescents in science, technology, engineering, mathematics, and medicine (STEMM) topics. The program is the first of its kind in Canada, and to our knowledge, internationally. Each session aims to foster health promotion and preventative medicine by providing interactive and fun presentations centering on science and technology. Concepts presented in previous shows include the signs and symptoms of common medical conditions (i.e. myocardial infarction, stroke, fractures), medical equipment (i.e. heart valves, catheters, and stents), basic cardiopulmonary resuscitation (CPR) skills, simulated medical procedures (i.e. bone internal fixation and intubation), and the biology of microscopic zebrafish larvae, amongst many other topics. Since its inception in May 2018, the series has relied on 3D-printed anatomical models that allow attendees to have hands-on exploration of structures related to the given presentation. The low-cost nature of these items permits some members of the audience to be gifted the models to take home with them. Those involved in Medical Curiosity on Stage hope that these outreach efforts provide young children with positive early experiences and foster enthusiasm to pursue future experiences in STEMM.

\section{FUTURE DEVELOPMENTS}

At the intersection of medicine and academia, the $\mathrm{TOH}$ 3D Printing Program is at the natural crossroads for the development and deployment of research innovations within the healthcare sector. Several innovations are currently in development and being assessed for translation to clinical patient care. Here we highlight a number of innovations being actively studied at the TOH 3D Printing Program.

\section{Arterial Phantoms}

3D-printed blood vessels are increasingly being used to visualize patient- and pathology-specific states in the field of cardiovascular surgery. Preoperatively, 3D-printed blood vessels can facilitate the selection and intended location of endovascular stents in complex aortic aneurysms and coarctations (16-18). Likewise, in other settings, 3D-printed blood vessels have been employed to aid surgical practice amongst medical trainees and to evaluate the utility of imaging technologies in endovascular interventions $(19,20)$. However, the degree to which these 3D-printed models recapitulate physiological characteristics of blood vessels has been limited thus far. At the TOH 3D Printing Program, research is underway to improve the fidelity and the utility of these so-called "arterial phantom" models for surgical planning and training.

\section{Surgical Guides}

In prosthodontics, 3D printing technology has been used to create patient-specific surgical guides. These structures, unlike patient-specific anatomical models, are designed to precisely overlay a patient's teeth and incorporate intentional gaps in material to indicate the desired location for needle passes or surgical drills. By this means, surgical procedures may be "templated", allowing the placement of dental implants with superior precision compared to conventional techniques (i.e., gypsum stone and denture models) (21). It is conceivable that this application of custom 3D printing be extended to the guidance of anesthetic injections or biopsy procedures in other medical fields. The feasibility of related applications is currently under investigation at the TOH 3D Printing Program. 


\section{Implantable Scaffolds for Bone Growth}

In Australia, a novel 3D-printed solution was devised for treatment of osteomyelitis-mediated tibial bone destruction (22). In such cases, traditional management would favor an above-knee amputation. This outcome was obviated by the transplantation of 3D-printed scaffold containing fibular bone tissue and blood supply grafted from the contralateral leg (22). While long-term growth and functional outcomes are not yet known in this patient case, the $\mathrm{TOH}$ 3D Printing Program is making very preliminary assessments regarding the viability of generating similar tissue-based scaffolds.

\section{CONCLUSION}

There is no doubt that $3 \mathrm{D}$ printing technology is revolutionizing healthcare. In this article, we have reviewed select major projects pioneered at the TOH 3D Printing Program, including those relating to surgical and medical education. We have also highlighted a number of putative future applications that could harness 3D printing technology to improve patient care in Ottawa. There are, however, limitations of 3D printing that must be acknowledged. Foremost of these limitations is the up-front cost of 3D printing materials and equipment, the attainment of personnel familiar with the technology, as well as the paucity of evidence demonstrating clinical efficacy of $3 \mathrm{D}$ printed devices in certain applications $(9,23)$. With ongoing research at the TOH 3D Printing Program, amongst other centers, we expect these limitations to be gradually minimized while clinically efficacious applications of 3D technology become progressively more prevalent.

\section{REFERENCES}

1. Hull C. Apparatus for production of three-dimensional object by stereolithography. US Pat A. 1986;4575330:1986.

2. Berman B. 3-D printing: The new industrial revolution. Bus Horiz. 2012;55(2):155-62.

3. Ventola CL. Medical Applications for 3D Printing: Current and Projected Uses. PT. 2014;39(10):704-11.

4. Schubert C, van Langeveld MC, Donoso LA. Innovations in 3D printing: a 3D overview from optics to organs. Br J Ophthalmol. 2014;98(2):159-61.

5. Michalski MH, Ross JS. The Shape of Things to Come. JAMA. 2014;312(21):2213.

6. Aimar A, Palermo A, Innocenti B. The Role of 3D Printing in Medical Applications: A State of the Art. J Healthc Eng. 2019;2019:1-10.

7. Markets and Markets. 3D Printing Medical Devices Market by Component (3D Printers, 3D Bioprinters, Material (Plastic, Metal, Ceramic), Software \& Services), Technology (EBM, LBM, Photopolymerization, 3DP, and DD), Product Type (Prosthetics, Implant) - Global Forecast to 2022 [Internet]. 2017 [cited 2019 Apr 11]. Available from: https://www.marketsandmarkets. com/Market-Reports/3d-printing-medical-devices-market-90799911. $\mathrm{html}$

8. 3D printing our way to better health care [Internet]. The Ottawa Hospital Foundation. 2017 [cited 2019 Apr 3]. Available from: https://www. ottawahospital.on.ca/en/youre-in-my-care/3d-printing-our-way-tobetter-health-care/
9. Tack P, Victor J, Gemmel P, Annemans L. 3D-printing techniques in a medical setting: a systematic literature review. Biomed Eng Online. 2016;15(1):115.

10. $3 \mathrm{D}$ printing helps complex surgery to remove 50 tumours, save uterus | The Ottawa Hospital Foundation [Internet]. The Ottawa Hospital Foundation. [cited 2019 Feb 28]. Available from: https://ohfoundation.ca/ our-supporters/3d-printing-helps-complex-surgery-remove-50-tumourssave-uterus

11. Flaxman T, Sheikh A, Althobaity W, Miguel O, Cooke C, Singh S. Optimizing Pre-surgical Planning for a Complex Myomectomy Using a PatientSpecific Three-Dimensional Printed Anatomical Model. J Obstet Gynaecol Can. 2019;0(0).

12. Fagerlin A, Lakhani I, Lantz PM, Janz NK, Morrow M, Schwartz K, et al. An informed decision? Patient Educ Couns. 2006;64(1-3):303-12.

13. Yiasemidou M, Gkaragkani E, Glassman D, Biyani CS. Cadaveric simulation: a review of reviews [Internet]. Vol. 187, Irish Journal of Medical Science. 2018. p. 827-33.

14. AbouHashem $Y$, Dayal M, Savanah S, Štrkalj G. The application of 3D printing in anatomy education. Med Educ Online. 2015;20(1):29847.

15. Canada Science and Technology Museum. The new Canada Science and Technology Museum welcomes over 650,000 visitors in its first year | Ingenium [Internet]. 2018 [cited 2019 Apr 1]. Available from: https://ingeniumcanada.org/newsroom/the-new-canada-science-andtechnology-museum-welcomes-over-650000-visitors-in-its-first

16. Malik HH, Darwood ARJ, Shaunak S, Kulatilake P, El-Hilly AA, Mulki O, et al. Three-dimensional printing in surgery: a review of current surgical applications. J Surg Res. 2015;199(2):512-22.

17. Biglino G, Capelli C, Taylor AM, Schievano S. 3D Printing Cardiovascular Anatomy: A Single-Centre Experience. In: New Trends in 3D Printing. 2016.

18. Tam MDBS, Laycock SD, Brown JRI, Jakeways M. 3D Printing of an Aortic Aneurysm to Facilitate Decision Making and Device Selection for Endovascular Aneurysm Repair in Complex Neck Anatomy. J Endovasc Ther. 2013;20(6):863-7.

19. Kimura T, Morita A, Nishimura K, Aiyama H, Itoh H, Fukaya S, et al. Simulation of and training for cerebral aneurysm clipping with 3-dimensional models. Neurosurgery. 2009;65(4):719-26.

20. Nagesh SVS, Hinaman J, Sommer K, Xiong Z, lonita CN, Bednarek DR, et al. A simulation platform using 3D printed neurovascular phantoms for clinical utility evaluation of new imaging technologies. Proc SPIE--the Int Soc Opt Eng. 2018;10578.

21. Ma B, Park T, Chun I, Yun K. The accuracy of a 3D printing surgical guide determined by $\mathrm{CBCT}$ and model analysis. J Adv Prosthodont. 2018;10(4):279-85.

22. Minion L. Queensland surgeons perform world-first 3D printed shinbone transplant | Healthcare IT Australia [Internet]. Healthcare Information and Management Systems Society. 2017 [cited 2019 Feb 24]. Available from: https://www.healthcareit.com.au/article/queensland-surgeons-performworld-first-3d-printed-shinbone-transplant

23. Diment LE, Thompson MS, Bergmann JHM. Clinical efficacy and effectiveness of 3D printing: a systematic review. BMJ Open. 2017;7(12):e016891. 